\title{
23. SUMMARY OF SHIPBOARD PHYSICAL PROPERTIES OF MUDSTONES, CARBONATES, AND CLASTIC SEDIMENTS, DEEP SEA DRILLING PROJECT, LEG 721
}

\author{
C. H. Schaftenaar, R. P. Moore, and R. L. Carlson, Department of Geophysics, Texas A\&M University, \\ College Station, Texas
}

\section{INTRODUCTION}

Compressional-wave velocity, wet-bulk density, and porosity were measured on sediments and rocks recovered from Deep Sea Drilling Project Holes 515B and 516F. Wet-bulk densities were measured by both gravimetric and GRAPE methods. Velocities were measured on trimmed samples with the Hamilton frame velocimeter. The shipboard measurement techniques are discussed in the explanatory notes chapter (Coulbourn, this volume) and are described in detail by Boyce (1976a). Only the shipboard measurements are reported here. The results of shore-based laboratory studies of the physical properties of the carbonates and basalts from Hole $516 \mathrm{~F}$ can be found in Carlson and others (this volume) and Gebhard and Carlson (this volume). Mechanical properties of the sediments are discussed in Walton and others (this volume) and Faas and Crocket (this volume).

The physical properties of 55 mudstone samples recovered from Hole 515B between sub-bottom depths of 256 and $635 \mathrm{~m}$ are summarized in Table 1. Extensive physical property measurements were made at Hole $516 \mathrm{~F}$ on samples from sub-bottom depths between 172 and $1231 \mathrm{~m}$, including 319 carbonates, 5 volcanic ashes and sandstones, and 1 mudstone. The physical properties are listed in Table 2.

\section{DENSITY-POROSITY RELATIONS}

Wet-bulk densities $\left(\varrho_{b}\right)$ of mudstones, carbonates, and clastic sediments were estimated by gravimetric (immersion) and 2-minute GRAPE methods as described by Boyce $(1976 a, b)$. "Gravimetric" porosities $(\phi)$ were calculated from the gravimetric densities, water contents $\left(W_{\mathrm{c}}\right)$, and pore-fluid density $\left(\varrho_{\mathrm{f}}=1.025 \mathrm{~g} / \mathrm{cm}^{3}\right)$ of the sediments:

$$
\phi=\varrho_{\mathrm{b}} W_{\mathrm{c}} / \varrho_{\mathrm{f}}
$$

GRAPE porosities were calculated from estimated bulk densities by assuming a mean-grain density $\left(\varrho_{g}\right)$ of $2.71 \mathrm{~g} / \mathrm{cm}^{3}$.

For suites of samples having the same mean-grain density, fractional porosity and wet-bulk density are linearly related:

$$
\varrho_{\mathrm{b}}=\varrho_{\mathrm{g}}-\phi\left(\varrho_{\mathrm{g}}-\varrho_{\mathrm{f}}\right) .
$$

\footnotetext{
${ }^{1}$ Barker, P. F., Carlson, R. L., Johnson, D. A., et al., Init, Repts. DSDP, 72: Washing ton (U.S. Govt. Printing Office).
}

Thus, both mean-grain and pore-fluid density can be estimated by linear regression if independent measurements of bulk density and fractional porosity have been made.

Gravimetric and GRAPE densities are plotted against gravimetric porosities of mudstones from Hole 515B in Figures 1 and 2, respectively. The implied pore-fluid density $\left(\varrho_{\mathrm{f}}=\mathrm{a}+\mathrm{b}\right.$; see Table 3$)$ of the mudstones is $0.86 \mathrm{~g} / \mathrm{cm}^{3}$ when estimated from the GRAPE density data and $0.92 \mathrm{~g} / \mathrm{cm}^{3}$ when estimated from gravimetric densities. These values are too low for fully saturated sediments, suggesting that these sediments may have been slightly undersaturated at the time of measurement (i.e., the gravimetric porosities calculated by $\phi=\varrho_{\mathrm{b}} W_{\mathrm{c}}$ ' $\varrho_{\mathrm{f}}$ are low because the water content, $W_{\mathrm{c}}$, was underestimated). Undersaturation could occur because of sample volume expansion upon release of overburden pressure and the subsequent uptake of air into the pore spaces or because of slight drying of the samples before measurement.

The mean-grain densities $\varrho_{\mathrm{g}}$ (parameter a in Table 3) of the mudstones are estimated to be $2.86 \mathrm{~g} / \mathrm{cm}^{3}$ from the gravimetric density data and $2.81 \mathrm{~g} / \mathrm{cm}^{3}$ from the GRAPE densities. Grain densities of clayey silts and silty clays, measured by the pycnometer method, have been reported to be as high as $2.79 \mathrm{~g} / \mathrm{cm}^{3}$ (Bachman and Hamilton, 1976). However, the grain density estimated for the mudstones from Hole 515B may be too large because there are two distinct groups of mudstone samples that have different apparent grain densities. Samples with porosities above $60 \%$ are siliceous mudstones that contain about $15 \%$ siliceous biogenic material. In contrast, the samples with porosities less than $55 \%$ generally contain no siliceous biogenic material but do contain small amounts of assorted heavy minerals. Thus, it is likely that the higher-porosity, siliceous mudstones have a relatively low mean-grain density, whereas the lower porosity mudstones have a relatively higher meangrain density. Therefore, the high grain densities and low pore-fluid densities estimated by linear regression for the complete data set could be an artifact of fitting the regression line through two groups of samples with different grain densities (i.e., the two groups should lie on separate $\varrho-\phi$ curves because they have different mean-grain densities).

Theoretical wet-bulk density-porosity relationships for completely saturated sediments with a pore-fluid density of $1.025 \mathrm{~g} / \mathrm{cm}^{3}$ are shown for comparison with the measured values from Hole 515B and the regression 
Table 1. Summary of physical properties for Hole 515B.

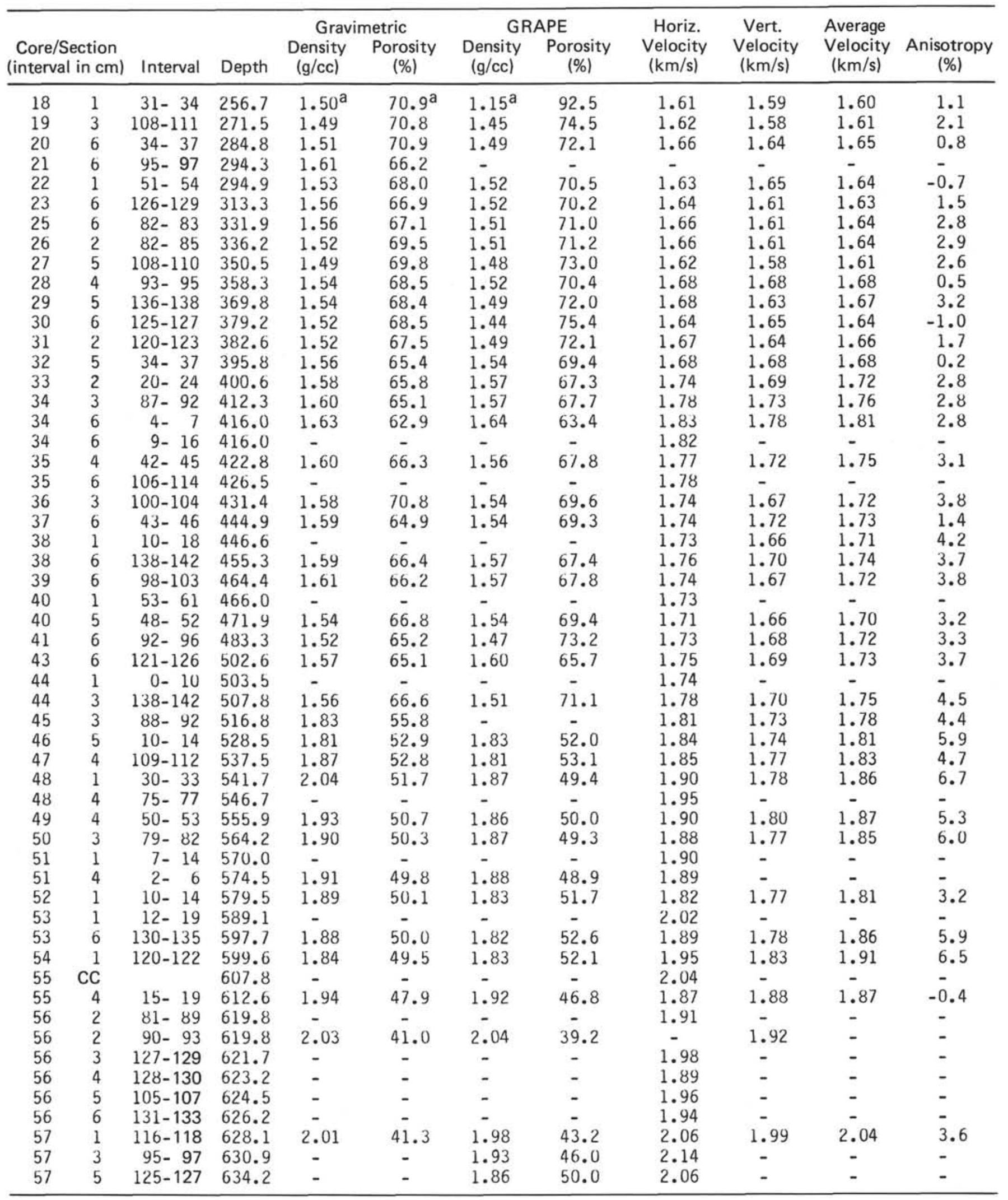

a These data were not used in the regressions shown in Figures 1 and 2 and 1 isted in Table 3 . 
Table 2. Summary of physical properties for Hole 516F.

\begin{tabular}{|c|c|c|c|c|c|c|c|c|c|c|c|}
\hline & & & & Grav & netric & & APE & Horiz. & Vert. & Average & \\
\hline $\begin{array}{r}\text { Core/S } \\
\text { (interva }\end{array}$ & $\begin{array}{l}\text { ection } \\
\text { in } \mathrm{cm} \text { ) }\end{array}$ & Interval & Depth & $\begin{array}{c}\text { Density } \\
\text { (g/cc) }\end{array}$ & $\begin{array}{c}\text { Porosity } \\
(\%)\end{array}$ & $\begin{array}{c}\text { Density } \\
(\mathrm{g} / \mathrm{cc})\end{array}$ & $\begin{array}{c}\text { Porosity } \\
(\%)\end{array}$ & $\begin{array}{l}\text { Velocity } \\
(\mathrm{km} / \mathrm{s})\end{array}$ & $\begin{array}{l}\text { Velocity } \\
(\mathrm{km} / \mathrm{s})\end{array}$ & $\begin{array}{l}\text { Velocity } \\
(\mathrm{km} / \mathrm{s})\end{array}$ & $\begin{array}{c}\text { Anisotropy } \\
(\%)\end{array}$ \\
\hline 1 & 2 & $92-94$ & 172.5 & - & - & - & - & - & - & - & - \\
\hline 1 & 2 & $95-97$ & 171.6 & - & - & - & - & 1.64 & - & - & - \\
\hline 1 & 2 & $100-102$ & 171.6 & - & - & - & - & - & - & - & - \\
\hline 1 & 2 & $103-105$ & 171.6 & - & - & - & - & - & - & - & - \\
\hline 2 & 7 & $21-23$ & 187.8 & 1.73 & 57.2 & 1.73 & 58.4 & 1.77 & 1.78 & 1.77 & -0.4 \\
\hline 5 & 6 & $107-109$ & 215.7 & 1.81 & 52.4 & 1.76 & 56.4 & 1.82 & 1.74 & 1.79 & 4.8 \\
\hline 6 & 1 & $90-93$ & 217.5 & 1.81 & 52.2 & 1.76 & 56.1 & 1.73 & 1.67 & 1.71 & 3.4 \\
\hline 7 & 3 & $33-35$ & 229.4 & 1.80 & 52.3 & 1.80 & 53.9 & 1.72 & 1.76 & 1.74 & -2.4 \\
\hline 8 & 6 & $76-80$ & 243.9 & 1.77 & 54.0 & 1.72 & 58.5 & 1.68 & 1.66 & 1.67 & 1.7 \\
\hline 9 & 4 & $72-74$ & 250.3 & 1.75 & 53.3 & 1.69 & 60.7 & 1.70 & 1.75 & 1.71 & -2.9 \\
\hline 10 & 4 & $1-\quad 5$ & 259.1 & 1.75 & 54.9 & 1.70 & 59.8 & 1.71 & 1.69 & 1.70 & 1.3 \\
\hline 11 & 1 & $0-11$ & 264.1 & - & - & - & - & 1.72 & - & - & - \\
\hline 11 & 3 & $80-83$ & 267.9 & 1.82 & 52.3 & 1.76 & 56.5 & 1.78 & 1.75 & 1.77 & 1.2 \\
\hline 12 & 1 & $12-15$ & 273.7 & $1.90^{\mathrm{a}}$ & 54.5 & 1.77 & 55.7 & 1.73 & 1.72 & 1.73 & 1.0 \\
\hline 16 & 1 & $0-5$ & 311.6 & 1.87 & 47.8 & 1.84 & 51.8 & 1.82 & 1.73 & 1.79 & 5.4 \\
\hline 17 & $\mathrm{CC}$ & & 324.2 & - & - & - & - & 1.71 & - & - & - \\
\hline 18 & $\mathrm{CC}$ & & 331.9 & 1.91 & 47.7 & 1.84 & 51.4 & 1.75 & 1.77 & 1.76 & -1.2 \\
\hline 19 & 2 & $75-84$ & 342.4 & $2.02^{a}$ & 49.2 & 1.95 & 44.9 & 1.88 & 1.83 & 1.86 & 2.5 \\
\hline 19 & 2 & $117-120$ & 342.8 & - & - & 1.89 & 48.5 & 2.07 & - & - & - \\
\hline 20 & 1 & $11-13$ & 349.7 & 1.96 & 44.1 & 1.95 & 45.4 & 1.89 & - & - & - \\
\hline 21 & 1 & $29-31$ & 359.4 & 1.95 & 44.7 & 1.93 & 46.3 & 1.94 & - & - & - \\
\hline 22 & 1 & $5-7$ & 368.7 & 1.95 & 45.6 & - & - & - & - & - & - \\
\hline 22 & 1 & $7-14$ & 368.7 & - & - & 1.91 & 47.2 & 2.04 & - & - & - \\
\hline 23 & 2 & $12-15$ & 379.7 & 1.95 & 44.8 & 1.96 & 44.5 & 1.91 & 1.88 & 1.90 & 1.6 \\
\hline 24 & 1 & $17-26$ & 387.8 & - & - & - & - & 1.88 & - & - & - \\
\hline 24 & 1 & $49-52$ & 388.1 & 1.98 & 43.5 & 2.00 & 42.0 & 1.94 & 1.87 & 1.91 & 3.7 \\
\hline 25 & 1 & $15-17$ & 397.3 & 2.01 & 45.1 & 1.96 & 44.3 & 1.77 & 1.78 & 1.77 & -0.4 \\
\hline 25 & 3 & $44-53$ & 400.6 & - & - & 1.98 & 43.2 & 1.87 & - & - & - \\
\hline 26 & 1 & $17-25$ & 406.8 & - & - & 2.02 & 40.8 & 1.97 & - & - & - \\
\hline 26 & 5 & $124-127$ & 413.9 & 2.02 & 41.8 & 2.03 & 40.6 & 1.89 & 1.84 & 1.87 & 2.6 \\
\hline 27 & 1 & 5- 8 & 416.2 & 1.96 & 48.3 & 1.91 & 47.6 & 1.79 & 1.77 & 1.79 & 1.0 \\
\hline 27 & 4 & $34-37$ & 421.0 & 1.95 & 45.0 & 1.95 & 45.4 & 1.89 & 1.82 & 1.87 & 3.5 \\
\hline 28 & 2 & $73-79$ & 427.9 & 1.87 & 48.5 & $1.70^{\mathrm{a}}$ & 59.8 & 1.76 & 1.72 & 1.75 & 2.2 \\
\hline 29 & 1 & $41-51$ & 435.6 & - & - & - & - & 1.91 & - & - & - \\
\hline 29 & 5 & $90-95$ & 442.0 & $2.22^{a}$ & 52.2 & 1.91 & 47.6 & 1.88 & 1.84 & 1.87 & 2.3 \\
\hline 30 & 2 & $142-145$ & 447.5 & $2.08^{a}$ & 46.2 & 1.91 & 47.2 & 1.91 & 1.88 & 1.90 & 1.9 \\
\hline 30 & 6 & $38-42$ & 452.5 & 1.96 & 44.7 & 1.92 & 46.9 & 1.93 & 1.85 & 1.90 & 3.9 \\
\hline 31 & 1 & $31-34$ & 454.4 & 1.96 & 45.0 & 2.00 & 42.3 & 1.81 & 1.80 & 1.80 & 0.6 \\
\hline 31 & 4 & $77-81$ & 459.4 & 2.02 & 41.0 & 2.03 & 40.1 & 1.97 & 1.87 & 1.94 & 5.5 \\
\hline 32 & 1 & $15-25$ & 463.8 & - & - & - & - & 1.96 & - & - & - \\
\hline 32 & 6 & $40-45$ & 471.5 & 1.99 & 43.3 & 1.99 & 42.8 & 2.03 & 1.96 & 2.01 & 3.3 \\
\hline 33 & 1 & $57-61$ & 473.7 & 1.95 & 44.7 & 1.94 & 45.4 & 1.85 & 1.82 & 1.84 & 1.7 \\
\hline 33 & 6 & $43-48$ & 481.1 & 1.98 & 43.1 & 1.93 & 46.5 & 1.94 & 1.89 & 1.92 & 2.7 \\
\hline 34 & 1 & $25-35$ & 482.9 & - & - & - & - & 1.85 & - & - & - \\
\hline 34 & 3 & $45-49$ & 486.1 & 2.13 & 38.8 & 2.10 & 36.2 & 2.14 & 2.03 & 2.11 & 5.4 \\
\hline 35 & 1 & $128-132$ & 493.4 & 2.06 & 37.9 & 2.02 & 41.1 & 1.90 & 1.94 & 1.91 & -2.2 \\
\hline 35 & 4 & $57-61$ & 497.2 & 1.99 & 43.0 & 1.91 & 47.6 & 1.89 & 1.84 & 1.87 & 3.0 \\
\hline 36 & 1 & $33-37$ & 502.0 & 1.94 & 45.4 & 1.89 & 48.9 & 1.91 & 1.79 & 1.87 & 6.4 \\
\hline 36 & 2 & $54-65$ & 503.7 & - & - & - & - & 2.01 & - & - & - \\
\hline 37 & 1 & $28-33$ & 511.4 & 1.92 & 46.1 & 1.91 & 47.6 & 1.79 & 1.75 & 1.78 & 2.6 \\
\hline 37 & 3 & $114-118$ & 515.3 & 2.07 & 38.3 & 2.09 & 36.6 & 2.05 & 1.91 & 2.00 & 7.1 \\
\hline 38 & 1 & $65-69$ & 521.3 & 1.94 & 42.9 & 1.93 & 46.1 & 1.78 & 1.70 & 1.76 & 4.8 \\
\hline 39 & 1 & $78-82$ & 530.9 & 2.07 & 38.7 & 2.04 & 39.8 & 2.06 & 1.93 & 2.02 & 6.6 \\
\hline 40 & 1 & $2-\quad 4$ & 539.6 & 1.96 & 42.3 & 1.96 & 44.6 & 1.88 & 1.85 & 1.87 & 1.9 \\
\hline 41 & 1 & $85-93$ & 550.0 & - & - & 1.99 & 42.8 & 2.02 & - & - & - \\
\hline 42 & 1 & $13-15$ & 558.7 & 2.02 & 40.0 & 1.97 & 43.7 & 1.93 & 1.97 & 1.94 & -2.0 \\
\hline 43 & 1 & $6-8$ & 568.2 & 2.00 & 42.0 & - & - & 1.87 & 1.87 & 1.87 & -0.1 \\
\hline 44 & 1 & $4-6$ & 577.7 & 1.97 & 43.0 & 1.94 & 45.6 & 1.85 & 1.91 & 1.87 & -3.1 \\
\hline 45 & 1 & $6-16$ & 587.2 & - & - & 1.96 & 44.4 & 1.92 & - & - & - \\
\hline
\end{tabular}


Table 2. (Continued).

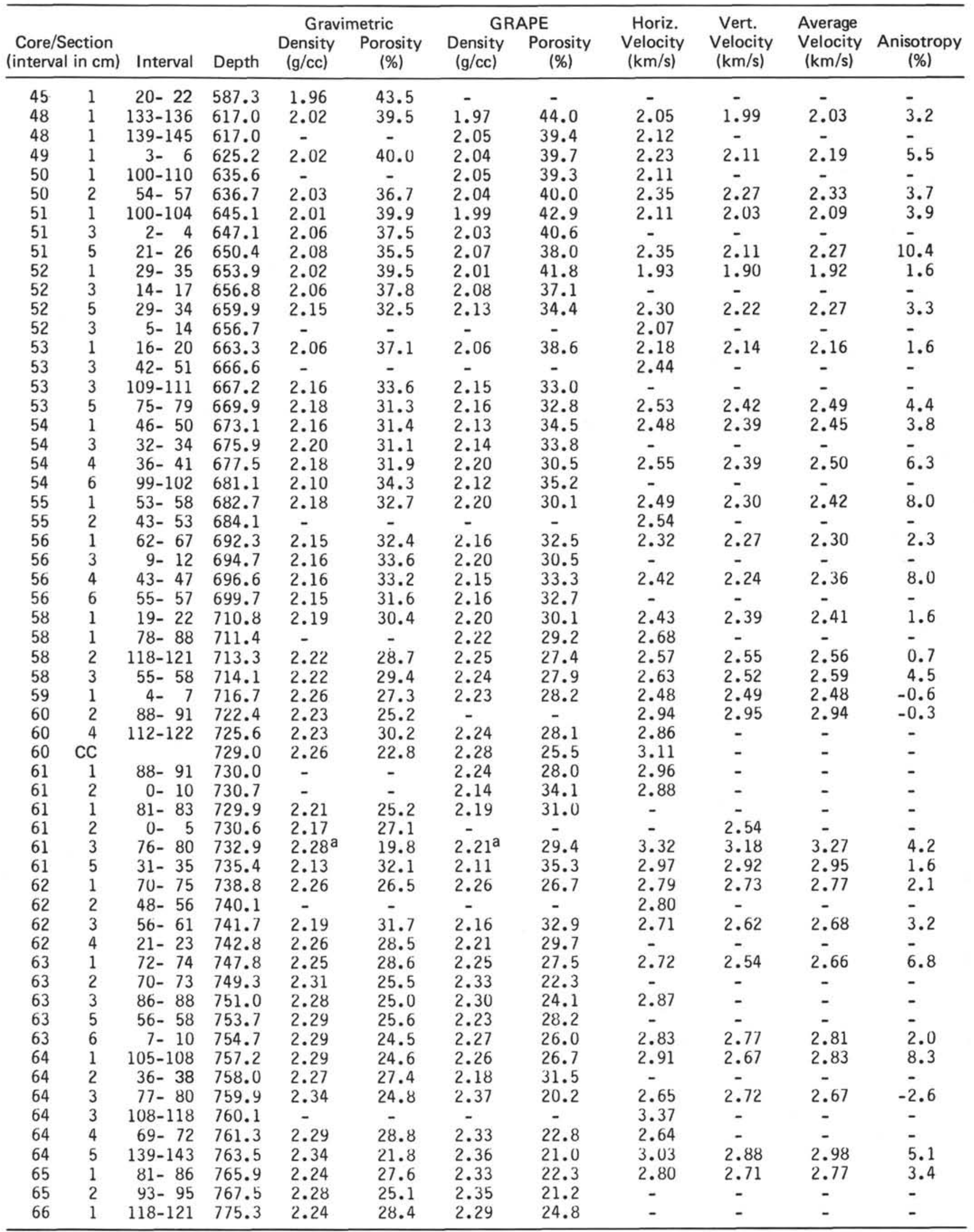


Table 2. (Continued).

\begin{tabular}{|c|c|c|c|c|c|c|c|c|c|c|c|}
\hline \multirow{2}{*}{\multicolumn{2}{|c|}{$\begin{array}{l}\text { Core/Section } \\
\text { (interval in cm) }\end{array}$}} & \multirow{3}{*}{$\frac{\text { Interval }}{122-132}$} & \multirow{3}{*}{$\begin{array}{l}\text { Depth } \\
775.4\end{array}$} & \multicolumn{2}{|c|}{ Gravimetric } & \multicolumn{2}{|c|}{ GRAPE } & \multirow{2}{*}{$\begin{array}{l}\text { Horiz. } \\
\text { Velocity } \\
(\mathrm{km} / \mathrm{s})\end{array}$} & \multirow{2}{*}{$\begin{array}{l}\text { Vert. } \\
\text { Velocity } \\
(\mathrm{km} / \mathrm{s})\end{array}$} & \multirow{2}{*}{$\begin{array}{l}\text { Average } \\
\text { Velocity } \\
(\mathrm{km} / \mathrm{s})\end{array}$} & \multirow[b]{2}{*}{$\begin{array}{c}\text { Anisotropy } \\
(\%)\end{array}$} \\
\hline & & & & $\begin{array}{l}\text { Density } \\
\text { (g/cc) }\end{array}$ & $\begin{array}{l}\text { Porosity } \\
\text { (\%) }\end{array}$ & $\begin{array}{l}\text { Density } \\
\text { (g/cc) }\end{array}$ & $\begin{array}{l}\text { Porosity } \\
\text { (\%) }\end{array}$ & & & & \\
\hline 66 & 1 & & & - & - & - & - & 2.86 & - & - & - \\
\hline 66 & 2 & $103-106$ & 776.6 & 2.28 & 25.4 & 2.30 & 24.4 & 2.86 & 2.71 & 2.81 & 5.3 \\
\hline 66 & 3 & $100-103$ & 778.1 & 2.27 & 26.6 & 2.28 & 25.8 & - & - & - & - \\
\hline 66 & 4 & $66-69$ & 779.3 & 2.26 & 27.0 & 2.20 & 30.4 & 2.88 & 2.68 & 2.81 & 7.1 \\
\hline 67 & 2 & $118-120$ & 784.3 & 2.24 & 28.8 & 2.24 & 27.9 & 2.79 & 2.67 & 2.75 & 4.5 \\
\hline 68 & 1 & $136-139$ & 787.5 & 2.43 & 19.1 & 2.43 & 16.8 & 3.42 & 3.39 & 3.41 & 0.8 \\
\hline 68 & 2 & $125-128$ & $788.9 \mathrm{~s}$ & $2.03^{a}$ & 45.9 & $2.05^{a}$ & 38.9 & 2.12 & 2.03 & 2.09 & 4.3 \\
\hline 68 & 4 & 9- 19 & 790.8 & 2.29 & 24.0 & 2.31 & 24.0 & 3.21 & - & - & - \\
\hline 69 & 1 & $47-50$ & 792.6 & 2.29 & 25.5 & 2.30 & 24.3 & - & 2.63 & - & - \\
\hline 69 & 2 & $130-132$ & 794.9 & 2.30 & 26.3 & 2.35 & 21.4 & 2.79 & 2.73 & 2.77 & 1.9 \\
\hline 70 & 1 & $22-32$ & 796.4 & 2.28 & 26.2 & 2.29 & 24.9 & 2.96 & - & - & - \\
\hline 70 & 1 & $61-64$ & 796.7 & 2.28 & 26.7 & 2.32 & 23.3 & 2.77 & 2.61 & 2.71 & 5.6 \\
\hline 71 & 1 & $113-117$ & 802.3 & 2.29 & 25.6 & 2.25 & 27.3 & 2.72 & 2.51 & 2.65 & 7.9 \\
\hline 71 & 2 & $70-72$ & 803.3 & 2.32 & 22.4 & 2.32 & 23.2 & 2.98 & 2.85 & 2.94 & 4.3 \\
\hline 71 & 3 & $86-89$ & 805.0 & 2.33 & 23. & 2.35 & 21.3 & 2.96 & 2.69 & 2.87 & 9.4 \\
\hline 71 & 4 & $85-87$ & 806.5 & 2.32 & 23.6 & 2.30 & 24.6 & - & - & - & - \\
\hline 71 & 4 & $88-91$ & 808.0 & 2.35 & 21.1 & 2.32 & 22.9 & 3.11 & 2.94 & 3.05 & 5.6 \\
\hline 72 & 1 & $48-50$ & 810.6 & 2.33 & 22.7 & 2.28 & 25.3 & 2.96 & 2.86 & 2.92 & 3.4 \\
\hline 72 & 2 & $4-14$ & 811.7 & - & - & - & - & 3.14 & - & - & - \\
\hline 72 & 2 & $55-57$ & 812.2 & - & - & 2.27 & 26.4 & 2.34 & - & - & - \\
\hline 72 & 3 & $61-65$ & 813.7 & 2.32 & 22.5 & 2.32 & 23.4 & 3.02 & 2.84 & 2.96 & 5.9 \\
\hline 72 & 4 & $43-46$ & $815.0 v$ & 2.35 & 21.6 & $2.57^{a}$ & 8.1 & - & - & - & - \\
\hline 73 & 1 & $65-68$ & 815.3 & 2.38 & 17.2 & 2.39 & 19.2 & 3.40 & 3.28 & 3.36 & 3.6 \\
\hline 73 & 2 & $117-120$ & 817.3 & 2.36 & 20.3 & 2.36 & 21.0 & 3.20 & - & - & - \\
\hline 73 & 3 & $120-123$ & 818.8 & 2.32 & 23.3 & 2.31 & 23.6 & 2.97 & 2.81 & 2.92 & 5.4 \\
\hline 73 & 4 & $57-60$ & 819.7 & 2.31 & 23.2 & 2.34 & 22.1 & 2.99 & - & - & - \\
\hline 73 & 5 & $68-70$ & 821.3 & - & - & - & - & 3.06 & 2.82 & 2.98 & 8.1 \\
\hline 74 & 1 & $117-119$ & 824.8 & 2.36 & 21.1 & 2.30 & 24.3 & 3.11 & 2.93 & 3.05 & 6.1 \\
\hline 74 & 2 & $111-113$ & 826.2 & 2.33 & 20.6 & 2.35 & 21.3 & 3.11 & - & - & - \\
\hline 74 & 3 & $81-83$ & 827.4 & 2.30 & 22.9 & 2.31 & 24.0 & 3.07 & 2.71 & 2.95 & 12.1 \\
\hline 74 & 4 & $140-143$ & 829.5 & 2.34 & 22.6 & 2.28 & 25.8 & 3.11 & - & - & - \\
\hline 74 & 5 & $40-42$ & 830.0 & 2.31 & 21.4 & 2.30 & 24.2 & 3.12 & 2.91 & 3.05 & 6.7 \\
\hline 74 & 6 & $68-70$ & 831.8 & 2.27 & 25.7 & 2.21 & 29.4 & 2.82 & - & - & - \\
\hline 75 & 1 & $89-91$ & 833.5 & $2.50^{a}$ & 21.4 & $2.47^{\mathrm{a}}$ & 14.1 & 3.08 & 3.02 & 3.06 & 1.9 \\
\hline 75 & 2 & $108-111$ & 835.2 & 2.29 & 24.5 & 2.28 & 25.5 & 2.92 & 2.76 & 2.87 & 5.8 \\
\hline 75 & 4 & $110-120$ & 838.3 & $2.14^{\mathrm{a}}$ & 16.3 & 2.39 & 18.7 & 3.36 & - & - & - \\
\hline 75 & 5 & $3-6$ & $838.7 \mathrm{~s}$ & $2.06^{a}$ & 44.9 & $2.15^{a}$ & 33.0 & 2.35 & 2.27 & 2.32 & 3.7 \\
\hline 75 & 5 & $84-86$ & 839.5 & $2.78^{a}$ & 14.9 & $2.82^{a}$ & - & 3.83 & 3.99 & 3.88 & -4.3 \\
\hline 75 & 6 & $143-145$ & 841.6 & 2.26 & 27.7 & 2.28 & 25.8 & 2.86 & 2.68 & 2.80 & 6.2 \\
\hline 76 & 2 & $123-126$ & $844.4 m$ & $2.14^{\mathrm{a}}$ & 33.8 & $2.12^{a}$ & 35.3 & 2.46 & 2.34 & 2.42 & 5.3 \\
\hline 76 & 4 & $116-126$ & $847.4 \mathrm{~s}$ & $1.94^{\mathrm{a}}$ & 49.1 & $1.96^{\mathrm{a}}$ & 44.3 & 2.20 & 2.12 & 2.17 & 3.7 \\
\hline 77 & 1 & $16-25$ & $850.8 s$ & - & - & 1.99 & 42.5 & 2.27 & 2.15 & 2.23 & 5.2 \\
\hline 77 & 2 & $93-95$ & 853.1 & 1.94 & 47.0 & 1.96 & 44.4 & 2.26 & 2.18 & 2.24 & 3.6 \\
\hline 77 & 4 & $73-75$ & 855.9 & 2.31 & 21.4 & 2.34 & 22.0 & 3.04 & 2.97 & 3.02 & 2.6 \\
\hline 78 & 1 & $71-74$ & 860.3 & 2.32 & 20.5 & 2.31 & 23.8 & 3.13 & 2.92 & 3.06 & 7.0 \\
\hline 78 & 2 & $133-136$ & 862.5 & 2.51 & 16.8 & 2.49 & 12.9 & 3.42 & 3.35 & 3.40 & 2.3 \\
\hline 78 & 4 & $1-11$ & 864.2 & - & - & 2.29 & 24.8 & 2.80 & - & - & - \\
\hline 78 & 4 & $120-123$ & 865.3 & 2.39 & 19.8 & 2.39 & 18.9 & 3.60 & 3.54 & 3.58 & 1.7 \\
\hline 79 & 1 & $30-32$ & 868.9 & 2.28 & 25.9 & 2.22 & 29.2 & 2.74 & 2.70 & 2.72 & 1.4 \\
\hline 79 & 2 & $29-31$ & 870.4 & 2.34 & 23.3 & 2.33 & 22.5 & 2.87 & 2.71 & 2.82 & 5.8 \\
\hline 79 & 3 & $100-102$ & 872.6 & 2.34 & 22.1 & 2.32 & 23.2 & 3.06 & 3.02 & 3.05 & 1.4 \\
\hline 79 & 4 & $93-95$ & 874.1 & 2.38 & 20.8 & 2.42 & 17.2 & 3.12 & 3.03 & 3.09 & 2.7 \\
\hline 79 & 2 & $103-93$ & 871.0 & - & - & 2.32 & 22.9 & 3.14 & - & - & - \\
\hline 79 & 5 & $70-73$ & 875.3 & 2.00 & 35.9 & 2.01 & 41.7 & 2.67 & 2.55 & 2.63 & 4.7 \\
\hline 80 & 1 & $113-115$ & 878.8 & 2.17 & 29.4 & 2.27 & 26.0 & 3.17 & 2.95 & 3.09 & 7.2 \\
\hline 80 & 2 & $108-110$ & 880.2 & 2.22 & 25.0 & 2.18 & 31.5 & 3.37 & 3.41 & 3.39 & -1.0 \\
\hline 80 & 2 & $32-45$ & 879.5 & - & - & 2.23 & 28.3 & 3.25 & - & - & - \\
\hline 81 & 1 & $104-106$ & 887.7 & 2.11 & 35.7 & 2.13 & 34.6 & 2.18 & 2.18 & 2.18 & -0.0 \\
\hline 81 & 2 & $106-108$ & 889.7 & 2.08 & 37.0 & 2.09 & 36.6 & 2.45 & 2.35 & 2.42 & 4.1 \\
\hline
\end{tabular}


Table 2. (Continued).

\begin{tabular}{|c|c|c|c|c|c|c|c|c|c|c|c|}
\hline \multirow{2}{*}{\multicolumn{2}{|c|}{$\begin{array}{l}\text { Core/Section } \\
\text { (interval in } \mathrm{cm} \text { ) }\end{array}$}} & \multirow{3}{*}{$\frac{\text { Interval }}{90-93}$} & \multirow{3}{*}{$\begin{array}{l}\text { Depth } \\
901.5\end{array}$} & \multicolumn{2}{|c|}{ Gravimetric } & \multicolumn{2}{|c|}{ GRAPE } & \multirow{2}{*}{$\begin{array}{l}\text { Horiz. } \\
\text { Velocity } \\
(\mathrm{km} / \mathrm{s})\end{array}$} & \multirow{2}{*}{$\begin{array}{l}\text { Vert. } \\
\text { Velocity } \\
(\mathrm{km} / \mathrm{s})\end{array}$} & \multirow{2}{*}{$\begin{array}{l}\text { Average } \\
\text { Velocity } \\
(\mathrm{km} / \mathrm{s})\end{array}$} & \multirow[b]{2}{*}{$\begin{array}{c}\text { Anisotropy } \\
(\%)\end{array}$} \\
\hline & & & & $\begin{array}{l}\text { Density } \\
(\mathrm{g} / \mathrm{cc})\end{array}$ & $\begin{array}{c}\text { Porosity } \\
(\%)\end{array}$ & $\begin{array}{c}\text { Density } \\
(\mathrm{g} / \mathrm{cc})\end{array}$ & $\begin{array}{c}\text { Porosity } \\
\text { (\%) }\end{array}$ & & & & \\
\hline 83 & 1 & & & 2.23 & 28.4 & 2.23 & 28.6 & 2.69 & 2.69 & 2.69 & 0.0 \\
\hline 83 & 2 & $80-82$ & 902.9 & 2.20 & 29.7 & 2.16 & 32.5 & 2.55 & 2.38 & 2.49 & 7.2 \\
\hline 83 & 4 & $22-24$ & 905.3 & 2.18 & 31.4 & 2.20 & 30.6 & 2.45 & 2.33 & 2.41 & 4.8 \\
\hline 83 & 5 & $29-31$ & 906.9 & 2.16 & 31.9 & 2.11 & 35.3 & 2.33 & - & - & - \\
\hline 83 & 6 & $41-43$ & 908.5 & 2.16 & 32.2 & 2.19 & 30.9 & 2.39 & 2.33 & 2.37 & 2.7 \\
\hline 84 & 1 & $46-48$ & 910.6 & 2.19 & 30.5 & 2.18 & 31.7 & 2.44 & 2.35 & 2.41 & 3.9 \\
\hline 84 & 2 & $62-64$ & 912.2 & 2.20 & 29.9 & 2.27 & 26.4 & 2.51 & 2.38 & 2.47 & 5.2 \\
\hline 84 & 3 & $35-37$ & 913.5 & 2.18 & 31.2 & 2.17 & 31.9 & 2.49 & 2.41 & 2.46 & 3.5 \\
\hline 84 & 4 & $21-23$ & 914.8 & 2.20 & 30.1 & 2.18 & 31.7 & 2.54 & 2.48 & 2.52 & 2.1 \\
\hline 84 & 5 & $135-137$ & 917.5 & 2.20 & 30.0 & 2.17 & 32.2 & 2.63 & 2.57 & 2.61 & 2.4 \\
\hline 84 & 6 & $20-30$ & 917.9 & - & - & 2.22 & 28.9 & 2.67 & - & - & - \\
\hline 85 & 1 & $29-32$ & 919.9 & 2.12 & 34.4 & 2.10 & 36.0 & 2.34 & 2.31 & 2.33 & 1.3 \\
\hline 85 & 2 & $28-38$ & 921.4 & - & - & 2.13 & 34.3 & 2.26 & - & - & - \\
\hline 86 & 1 & $55-58$ & 929.7 & 2.26 & 27.1 & 2.22 & 28.8 & 2.54 & 2.39 & 2.49 & 6.2 \\
\hline 86 & 2 & $44-54$ & 931.1 & - & - & 2.24 & 28.1 & 2.58 & - & - & - \\
\hline 86 & 3 & $128-130$ & 933.4 & 2.21 & 29.9 & 2.21 & 29.5 & 2.06 & 2.40 & 2.17 & -15.6 \\
\hline 86 & 5 & $7-9$ & 935.2 & 2.19 & 31.0 & 2.15 & 33.5 & 2.41 & 2.33 & 2.38 & 3.3 \\
\hline 86 & 7 & $17-19$ & 938.3 & 2.17 & 31.8 & 2.09 & 36.6 & 2.27 & 2.21 & 2.25 & 3.0 \\
\hline 87 & 1 & $54-57$ & 939.2 & 2.17 & 32.2 & 2.21 & 29.7 & 2.43 & 2.32 & 2.39 & 4.5 \\
\hline 87 & 2 & $140-143$ & 941.5 & $2.12^{\mathrm{a}}$ & 27.6 & 2.23 & 28.6 & 2.49 & 2.42 & 2.47 & 2.7 \\
\hline 87 & 3 & $131-134$ & 942.9 & 2.25 & 27.7 & 2.27 & 25.9 & 2.59 & 2.68 & 2.62 & -3.4 \\
\hline 87 & 4 & $144-147$ & 944.6 & 2.21 & 30.1 & 2.21 & 29.9 & 2.49 & 2.42 & 2.47 & 2.9 \\
\hline 87 & 5 & $112-121$ & 945.8 & - & - & 2.21 & 29.4 & 2.66 & - & - & - \\
\hline 87 & 7 & $0-3$ & 947.6 & 2.25 & 27.7 & 2.27 & 25.9 & 2.50 & 2.43 & 2.48 & 3.0 \\
\hline 88 & 2 & $3-6$ & 948.1 & 2.18 & 31.5 & 2.18 & 31.5 & 2.36 & 2.37 & 2.36 & -0.5 \\
\hline 88 & 2 & $2-5$ & 949.7 & 2.24 & 27.9 & 2.24 & 27.6 & 2.59 & 2.58 & 2.59 & 0.5 \\
\hline 89 & 1 & $42-45$ & 958.1 & 2.30 & 24.7 & 2.31 & 23.8 & 2.67 & 2.56 & 2.63 & 4.4 \\
\hline 89 & 3 & $88-91$ & 961.5 & 2.32 & 23.5 & $2.69^{a}$ & 1.2 & 2.78 & 2.63 & 2.73 & 5.5 \\
\hline 89 & 4 & $140-149$ & 963.6 & - & - & 2.29 & 24.8 & 3.16 & - & - & - \\
\hline 89 & 5 & $9-12$ & 963.7 & 2.37 & 20.5 & 2.39 & 19.0 & 3.29 & 3.20 & 3.26 & 2.7 \\
\hline 90 & 1 & $5-8$ & 967.2 & 2.21 & 30.1 & 2.20 & 30.2 & 2.37 & 2.27 & 2.34 & 4.4 \\
\hline 90 & 3 & $123-132$ & 971.4 & - & - & 2.15 & 33.4 & 2.48 & - & - & - \\
\hline 90 & 4 & $20-23$ & 971.8 & 2.13 & 34.3 & 2.13 & 34.2 & 2.26 & 2.21 & 2.24 & 2.3 \\
\hline 90 & 6 & $3-6$ & 974.7 & 2.17 & 32.7 & 2.11 & 35.6 & 2.42 & 2.44 & 2.43 & -0.7 \\
\hline 91 & 2 & $3-6$ & 978.2 & 2.23 & 28.6 & 2.24 & 27.8 & 2.50 & 2.39 & 2.46 & 4.4 \\
\hline 91 & 3 & $138-141$ & 980.5 & 2.25 & 27.9 & 2.27 & 26.3 & 2.50 & 2.40 & 2.46 & 4.0 \\
\hline 91 & 5 & $78-87$ & 983.4 & - & - & 2.27 & 26.3 & 2.58 & - & - & - \\
\hline 91 & 5 & $3-6$ & 984.2 & 2.29 & 25.8 & 2.31 & 24.0 & 2.41 & 2.34 & 2.39 & 3.1 \\
\hline 92 & 1 & $126-128$ & 987.4 & 2.18 & 31.3 & 2.17 & 32.3 & 2.45 & 2.30 & 2.40 & 6.1 \\
\hline 92 & 2 & $145-147$ & 989.1 & 2.19 & 31.4 & 2.13 & 34.4 & 2.25 & 2.17 & 2.22 & 3.6 \\
\hline 92 & 3 & $94-96$ & 990.1 & 2.24 & 28.2 & 2.23 & 28.3 & 2.41 & 2.35 & 2.39 & 2.6 \\
\hline 92 & 4 & $21-31$ & 990.9 & - & - & 2.35 & 21.5 & 2.59 & - & - & - \\
\hline 93 & 1 & $129-131$ & 996.9 & 2.27 & 25.6 & 2.23 & 28.3 & 2.58 & 2.45 & 2.54 & 5.2 \\
\hline 93 & 2 & $129-131$ & 998.4 & 2.22 & 29.5 & 2.21 & 29.8 & 2.42 & 2.32 & 2.38 & 3.9 \\
\hline 93 & 3 & $124-126$ & 999.9 & 2.22 & 28.6 & 2.24 & 28.1 & 2.47 & 2.35 & 2.43 & 4.8 \\
\hline 93 & 4 & $126-135$ & 1001.4 & - & - & 2.35 & 21.4 & 2.79 & - & - & - \\
\hline 93 & 5 & $130-132$ & 1002.9 & 2.33 & 23.7 & 2.34 & 22.2 & 2.71 & 2.51 & 2.64 & 7.6 \\
\hline 94 & 1 & $119-121$ & 1006.3 & 2.34 & 23.4 & 2.30 & 24.3 & 2.72 & 2.58 & 2.68 & 5.2 \\
\hline 94 & 3 & $38-40$ & 1008.5 & 2.35 & 22.6 & 2.29 & 25.2 & 2.72 & 2.52 & 2.65 & 7.6 \\
\hline 94 & 4 & $141-143$ & 1011.0 & 2.37 & 21.2 & 2.39 & 18.8 & 2.87 & 2.66 & 2.80 & 7.7 \\
\hline 94 & 6 & $2-12$ & 1012.7 & - & - & 2.32 & 23.1 & 2.83 & - & - & - \\
\hline 95 & 1 & $63-65$ & 1015.2 & 2.37 & 23.7 & 2.37 & 20.1 & 2.62 & 2.48 & 2.58 & 5.3 \\
\hline 95 & 3 & $12-14$ & 1017.7 & 2.36 & 23.3 & 2.34 & 22.2 & 2.64 & 2.47 & 2.58 & 6.7 \\
\hline 95 & 4 & $6-16$ & 1019.2 & - & - & 2.35 & 21.5 & 2.60 & - & - & - \\
\hline 95 & 5 & $17-20$ & 1020.8 & 2.36 & 21.8 & 2.36 & 20.6 & 2.64 & 2.53 & 2.60 & 4.0 \\
\hline 96 & 1 & $29-38$ & 1023.9 & - & - & 2.36 & 20.5 & 2.75 & - & - & - \\
\hline 96 & 2 & $35-37$ & 1025.5 & 2.34 & 21.9 & 2.32 & 23.3 & 2.80 & 2.68 & 2.76 & 4.6 \\
\hline 96 & 3 & $25-27$ & 1026.9 & 2.34 & 22.6 & 2.38 & 19.9 & 2.81 & 2.67 & 2.76 & 5.2 \\
\hline 97 & 1 & $36-39$ & 1030.0 & 2.31 & 24.1 & 2.34 & 21.9 & 2.68 & 2.55 & 2.63 & 4.9 \\
\hline
\end{tabular}


Table 2. (Continued).

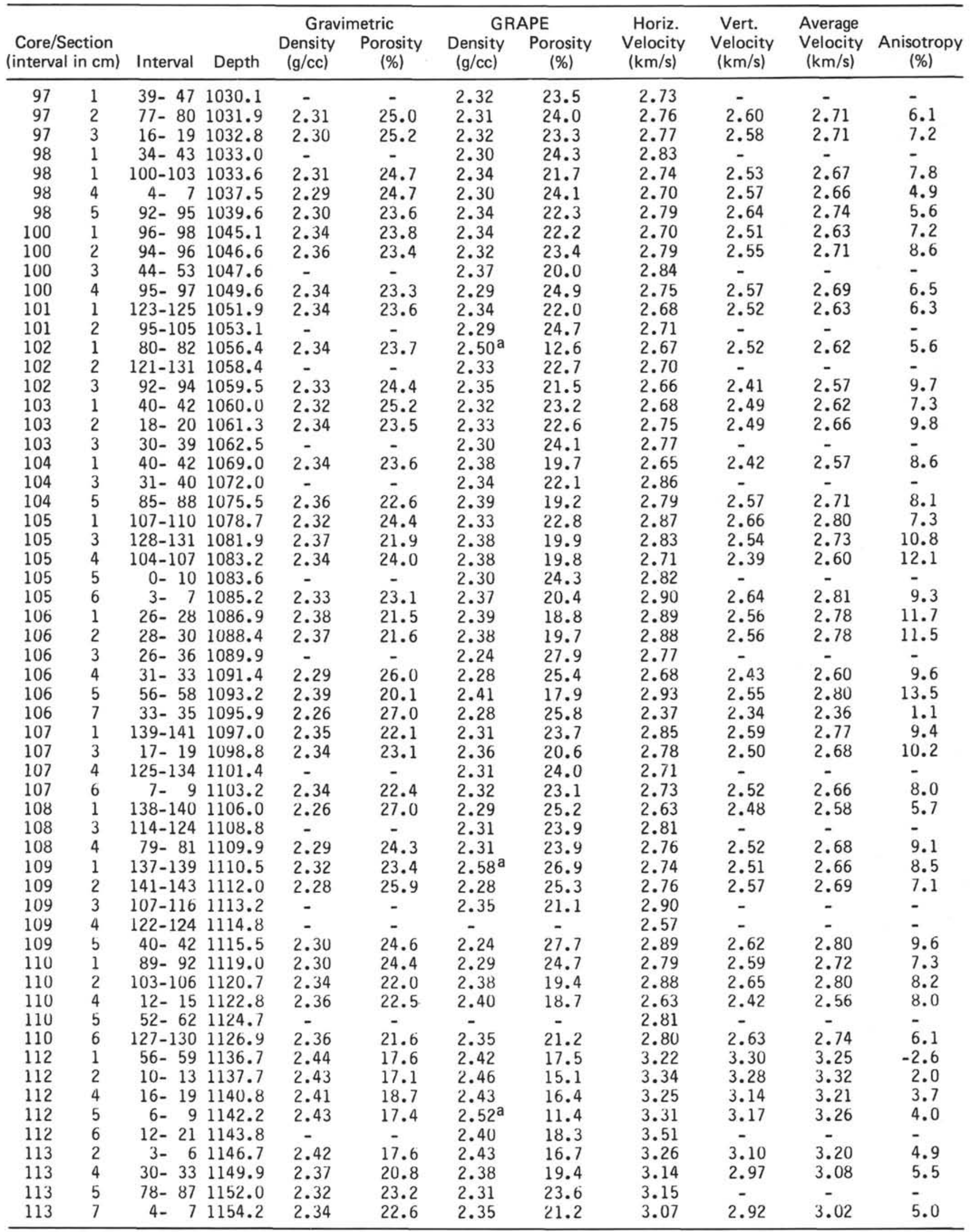


Table 2. (Continued).

\begin{tabular}{|c|c|c|c|c|c|c|c|c|c|c|c|}
\hline \multirow{2}{*}{\multicolumn{2}{|c|}{$\begin{array}{l}\text { Core/Section } \\
\text { (interval in cm) }\end{array}$}} & \multirow{3}{*}{$\begin{array}{l}\text { Interval } \\
26-36\end{array}$} & \multirow[b]{2}{*}{ Depth } & \multicolumn{2}{|c|}{ Gravimetric } & \multicolumn{2}{|c|}{ GRAPE } & \multirow{2}{*}{$\begin{array}{l}\text { Horiz. } \\
\text { Velocity } \\
(\mathrm{km} / \mathrm{s})\end{array}$} & \multirow{2}{*}{$\begin{array}{l}\text { Vert. } \\
\text { Velocity } \\
(\mathrm{km} / \mathrm{s})\end{array}$} & \multirow{2}{*}{$\begin{array}{l}\text { Average } \\
\text { Velocity } \\
(\mathrm{km} / \mathrm{s})\end{array}$} & \multirow[b]{2}{*}{$\begin{array}{c}\text { Anisotropy } \\
(\%)\end{array}$} \\
\hline & & & & $\begin{array}{l}\text { Density } \\
\text { (g/cc) }\end{array}$ & $\begin{array}{l}\text { Porosity } \\
\text { (\%) }\end{array}$ & $\begin{array}{l}\text { Density } \\
\text { (g/cc) }\end{array}$ & $\begin{array}{c}\text { Porosity } \\
\text { (\%) }\end{array}$ & & & & \\
\hline 114 & 1 & & 1154.4 & - & - & 2.35 & 21.5 & 3.41 & - & - & - \\
\hline 114 & 2 & $143-145$ & 1157.0 & 2.33 & 22.9 & 2.27 & 26.3 & 3.09 & 2.79 & 2.99 & 10.0 \\
\hline 114 & 4 & $13-15$ & 1158.7 & 2.40 & 18.2 & 2.41 & 18.0 & 3.45 & 3.20 & 3.37 & 7.3 \\
\hline 114 & 6 & $26-28$ & 1161.9 & 2.42 & 16.8 & 2.35 & 21.4 & 3.23 & 3.07 & 3.17 & 4.8 \\
\hline 115 & 1 & $113-115$ & 1164.2 & 2.41 & 17.2 & 2.40 & 18.2 & 3.36 & 3.06 & 3.26 & 9.4 \\
\hline 115 & 2 & $39-49$ & 1165.0 & - & - & 2.36 & 20.9 & 3.41 & - & - & - \\
\hline 115 & 3 & $72-74$ & 1166.8 & 2.40 & 17.8 & 2.37 & 20.1 & 3.35 & - & - & - \\
\hline 115 & 4 & $88-90$ & 1168.5 & 2.38 & 19.8 & 2.34 & 22.0 & 3.25 & 3.06 & 3.18 & 5.8 \\
\hline 116 & 1 & $32-34$ & 1172.4 & 2.41 & 17.6 & 2.40 & 18.2 & 3.55 & 3.24 & 3.45 & 9.2 \\
\hline 116 & 2 & $110-120$ & 1174.8 & - & - & 2.35 & 21.5 & 3.31 & - & - & - \\
\hline 116 & 4 & $21-23$ & 1176.8 & 2.45 & 15.2 & 2.45 & 15.7 & 3.81 & 3.63 & 3.75 & 4.7 \\
\hline 116 & 6 & $59-61$ & 1180.2 & 2.51 & 12.3 & 2.53 & 10.9 & 3.76 & 3.66 & 3.72 & 2.6 \\
\hline 117 & 1 & $40-43$ & 1181.5 & 2.48 & 13.5 & 2.46 & 14.9 & 3.57 & 3.28 & 3.47 & 8.4 \\
\hline 117 & 3 & $9-12$ & 1184.2 & 2.49 & 13.1 & 2.50 & 12.3 & 3.70 & 3.51 & 3.63 & 5.1 \\
\hline 117 & 4 & $115-125$ & 1186.8 & 2.46 & 15.7 & 2.46 & 14.7 & 3.67 & - & - & - \\
\hline 117 & 6 & $24-27$ & 1188.9 & 2.49 & 12.7 & 2.51 & 11.9 & 3.69 & 3.57 & 3.65 & 3.2 \\
\hline 118 & 1 & $141-144$ & 1192.5 & 2.51 & 12.5 & 2.49 & 13.1 & 3.71 & 3.43 & 3.62 & 7.6 \\
\hline 118 & 2 & $75-84$ & 1193.4 & - & - & 2.51 & 12.0 & 3.60 & - & - & - \\
\hline 118 & 3 & $97-100$ & 1195.1 & 2.49 & 13.4 & 2.50 & 12.7 & 3.87 & 3.45 & 3.73 & 11.2 \\
\hline 119 & 1 & $124-126$ & 1195.9 & 2.50 & 12.2 & 2.51 & 11.7 & 3.75 & 3.55 & 3.68 & 5.6 \\
\hline 119 & 2 & $127-129$ & 1197.4 & 2.48 & 13.4 & 2.50 & 12.5 & 3.90 & 3.64 & 3.82 & 6.8 \\
\hline 119 & 3 & $62-71$ & 1198.3 & - & - & 2.39 & 19.3 & 3.50 & - & - & - \\
\hline 119 & 4 & $123-125$ & 1200.4 & 2.48 & 15.1 & 2.49 & 13.0 & 3.42 & 3.16 & 3.33 & 7.8 \\
\hline 120 & 1 & $49-51$ & 1204.1 & 2.36 & 21.5 & 2.34 & 21.8 & 2.99 & 2.68 & 2.88 & 10.8 \\
\hline 120 & 2 & $33-35$ & 1205.5 & 2.27 & 25.2 & 2.24 & 27.9 & 3.02 & 2.56 & 2.87 & 16.0 \\
\hline 121 & 1 & $19-21$ & 1212.8 & 2.39 & 18.8 & 2.36 & 20.9 & 3.31 & 3.17 & 3.26 & 4.3 \\
\hline 122 & 1 & $77-86$ & 1222.4 & - & - & 2.45 & $15: 2$ & 4.36 & - & - & - \\
\hline 122 & 1 & $146-148$ & 1223.0 & 2.43 & 18.3 & 2.44 & 16.0 & 2.97 & 2.70 & 2.88 & 9.4 \\
\hline 122 & 2 & $89-91$ & 1224.0 & 2.36 & 19.7 & $2.21^{\mathrm{a}}$ & 29.9 & 3.55 & 3.13 & 3.41 & 12.2 \\
\hline 123 & 1 & $38-40$ & 1231.0 & 2.45 & 19.9 & 2.39 & 18.9 & 4.30 & 4.28 & 4.30 & 0.5 \\
\hline
\end{tabular}

a These data were not used in the regressions shown in Figures 3,4 , and 5 and 1 isted in Table 3.

Mudstones and clastics are indicated as follows: s, sandstone; m, mudstone; $v$, volcanic ash.

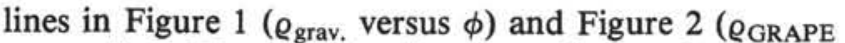
versus $\phi)$. In Figure 1, the siliceous mudstones $(\phi>60 \%)$ plot close to the line for the grain density of $2.6 \mathrm{~g} / \mathrm{cm}^{3}$, whereas the lower porosity mudstones plot closer to the line representing a grain density of $2.8 \mathrm{~g} / \mathrm{cm}^{3}$. In Figure 2 , the siliceous mudstones also plot close to the $2.6 \mathrm{~g} / \mathrm{cm}^{3}$ grain density line, but the lower porosity mudstones plot closer to the theoretical relationship with a grain density of $2.7 \mathrm{~g} / \mathrm{cm}^{3}$. Comparison of Figures 1 and 2 also show that the GRAPE densities are systematically lower than the gravimetric density values.

The density-porosity relationships for the carbonates recovered at Hole 516F are shown in Figures 3 and 4 for the gravimetric and GRAPE densities, respectively. Neither questionable data nor data for mudstones or clastic sediments were included in the regression analysis. Comparison of the figures shows that the scatter of the GRAPE data is larger than the scatter of the corresponding gravimetric density data. Mean-grain densities (see Table 3) and pore-fluid densities estimated by linear regression from the gravimetric density data are in good agreement with the expected values for carbonates (e.g.,
Hamilton, 1970; Bachman and Hamilton 1976; Hamilton, 1974).

\section{VELOCITY ANISOTROPY}

Deep-sea sediments usually exhibit transverse compressional-wave velocity isotropy and velocities parallel to bedding $\left(\mathrm{V}_{\mathrm{h}}\right)$ that are higher than velocities normal to bedding $\left(\mathrm{V}_{\mathrm{v}}\right)$ (Boyce, 1976a; Carlson and Christensen, 1979; Bachman, 1979; Carlson et al., this volume). When both horizontal and vertical compressional-wave velocities are available, transverse isotropy was assumed and the mean velocity $(\bar{V})$ and anisotropy $(A)$ were calculated by:

$$
\begin{aligned}
& \bar{V}=\left(2 V_{\mathrm{h}}+V_{\mathrm{v}}\right) / 3 \\
& A=100\left(V_{\mathrm{h}}-V_{\mathrm{v}}\right) / \bar{V} .
\end{aligned}
$$

The mean velocities and anisotropies calculated from the shipboard velocity data are shown in Tables 1 and 2 .

Anisotropies of the mudstones range from -1 to almost $7 \%$. The carbonates from Hole 516F have anisot- 


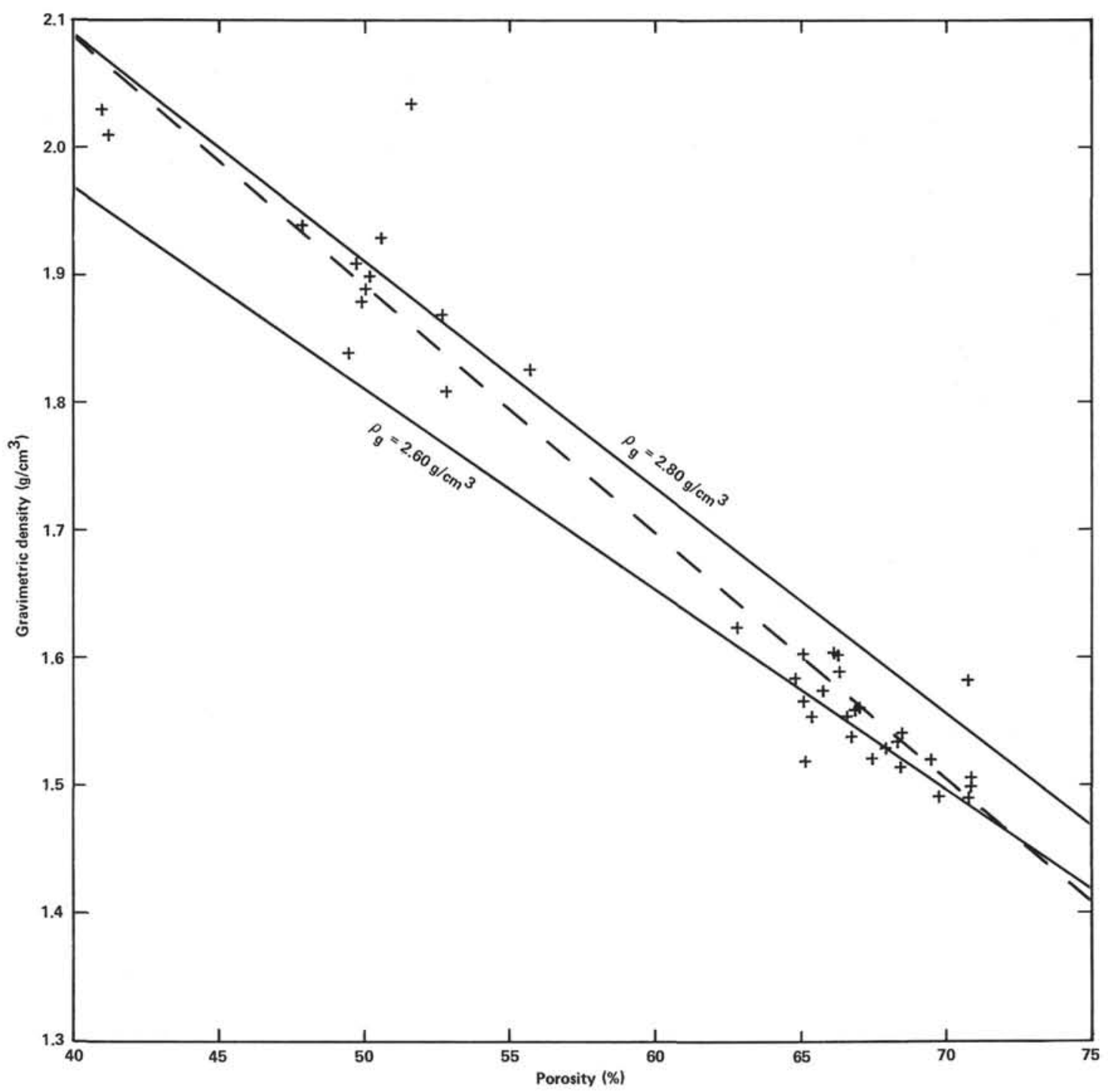

Figure 1. Hole 515B, gravimetric wet-bulk density versus gravimetric porosity. The dashed line shows the best-fitting linear relation determined by least squares (see Table 3 ). The solid lines show the theoretical relationships for completely saturated sediments. $\varrho_{\mathrm{b}}=\varrho_{\mathrm{f}} \phi+(1-\phi) \varrho_{\mathrm{g}}$ with a pore-fluid density of $1.025 \mathrm{~g} / \mathrm{cm}^{3}$ and grain densities as indicated.

ropies that, with the exception of Section 516F-86-3, range from -4.3 to $16 \%$. An error in one or both of the velocity measurements on this section was probably the cause of the large negative anisotropy of this sample $(A=-15.6 \%)$. Approximately $6 \%$ of the shipboard measurements indicate a negative anisotropy (i.e., $V_{\mathrm{v}}$ higher than $V_{\mathrm{h}}$ ). However, negative anisotropy is rarely observed in shore-based laboratory measurements (e.g., Carlson and Christensen, 1979; Carlson, 1982; Carlson, 1981; Carlson et al., this volume) and those that do occur are usually less negative than the uncertainty of the measurement. Most of the negative anisotropies reported are small, suggesting that errors in velocity measurements could produce them. For example, it is likely that trimmed surfaces on some samples are not parallel, in which case the measured velocity would be too low because the acoustic path is partially through water. Thus, measurements for samples that are isotropic or have a small positive anisotropy could show negative anisotropies.

\section{VELOCITY-DENSITY RELATIONS}

Figure 5 shows the average compressional-wave velocity plotted as a function of the gravimetric wet-bulk density for the carbonates from Hole $516 \mathrm{~F}$. The dashed line represents a nonlinear least-squares fit of $\varrho_{b}$ on $\bar{V}$. Questionable density data, which are indicated in Table 2 and Figure 5, were not included in the analysis. The regression parameters are shown in Table 3. For comparison, the empirical relationships determined by Hamilton (1978) and Milholland (1980) from laboratory measurements of velocity and density of calcareous deep-sea sediments are included in Figure 5. The shipboard measurements on the carbonates from Hole $516 \mathrm{~F}$ agree well with the empirical velocity-density curve of Hamilton 
(1978) but are systematically lower than the empirical curve of Milholland (1978).

\section{ACKNOWLEDGMENTS}

This research was supported by the Office of Naval Research Contract No. 0014-80-C-0113.

\section{REFERENCES}

Bachman, R. T., 1979. Acoustic anisotropy in marine sediments and sedimentary rocks. J. Geophys. Res., 84(B13):7661-7663.

Bachman, R. T., and E. L. Hamilton, 1976. Density, porosity, and grain density of samples from Deep Sea Drilling Project Site 222 (Leg 23) in the Arabian Sea. J. Sediment. Petrol., 46(3):654-658.

Boyce, R. E., 1976a. Definitions and laboratory techniques of compressional sound velocity parameters and wet-water content, wetbulk density, and porosity parameters by gravimetric and gamma ray attenuation techniques. In Schlanger, S. O., Jackson, E. D., et al., Init. Repts. DSDP, 33: Washington (U.S. Govt. Printing Office), 931-958.

1976b. Sound velocity-density parameters of sediment and rock from DSDP Drill Sites $315-318$ on the Line Islands Chain, Manihiki Plateau, and Tuamoto Ridge in the Pacific Ocean. In
Schlanger, S. O., Jackson, E. D., et al., Init. Repts. DSDP, 33: Washington (U.S. Govt. Printing Office), 695-728.

Carlson, R. L., 1981. Acoustic properties of limestones from the Northcentral Pacific, Deep Sea Drilling Project Leg 62. In Thiede, J., Vallier, T. L., et al., Init. Repts. DSDP, 62: Washington (U.S. Govt. Printing Office), 999-1004.

1982. Acoustic properties of tuffaceous and calcareous sediments, Deep Sea Drilling Project Leg 60. In Hussong, D. M., Uyeda, S., et al., Init. Repts. DSDP, 60: Washington (U.S. Govt. Printing Office), 803-804.

Carlson, R. L., and Christensen, N. I., 1979. Velocity anisotropy in semiindurated calcareous deep sea sediments. J. Geophys. Res., 84(B1):205-211.

Hamilton, E. L., 1970. Sound velocity and related properties of marine sediments, North Pacific. J. Geophys. Res., 75(23):4423-4446. 1974. Prediction of deep-sea sediment properties; state-ofthe-art. In Inderbitzen, A. L. (Ed.), Deep-Sea Sediments: Physical and Mechanical Properties: New York (Plenum Press), pp. 1-43. 1978. Sound velocity-density relations in sea-floor sediments and rocks. J. Acoust. Soc. Am., 63(2):366-377.

Milholland, P., Manghnani, M. H., Schlanger, S. O., and Sutton, G. H., 1980. Geoacoustic modeling of deep-sea carbonate sediments. J. Acoust. Soc. Am., 68(5):1351-1360.

Date of Initial Receipt: August 10, 1982 


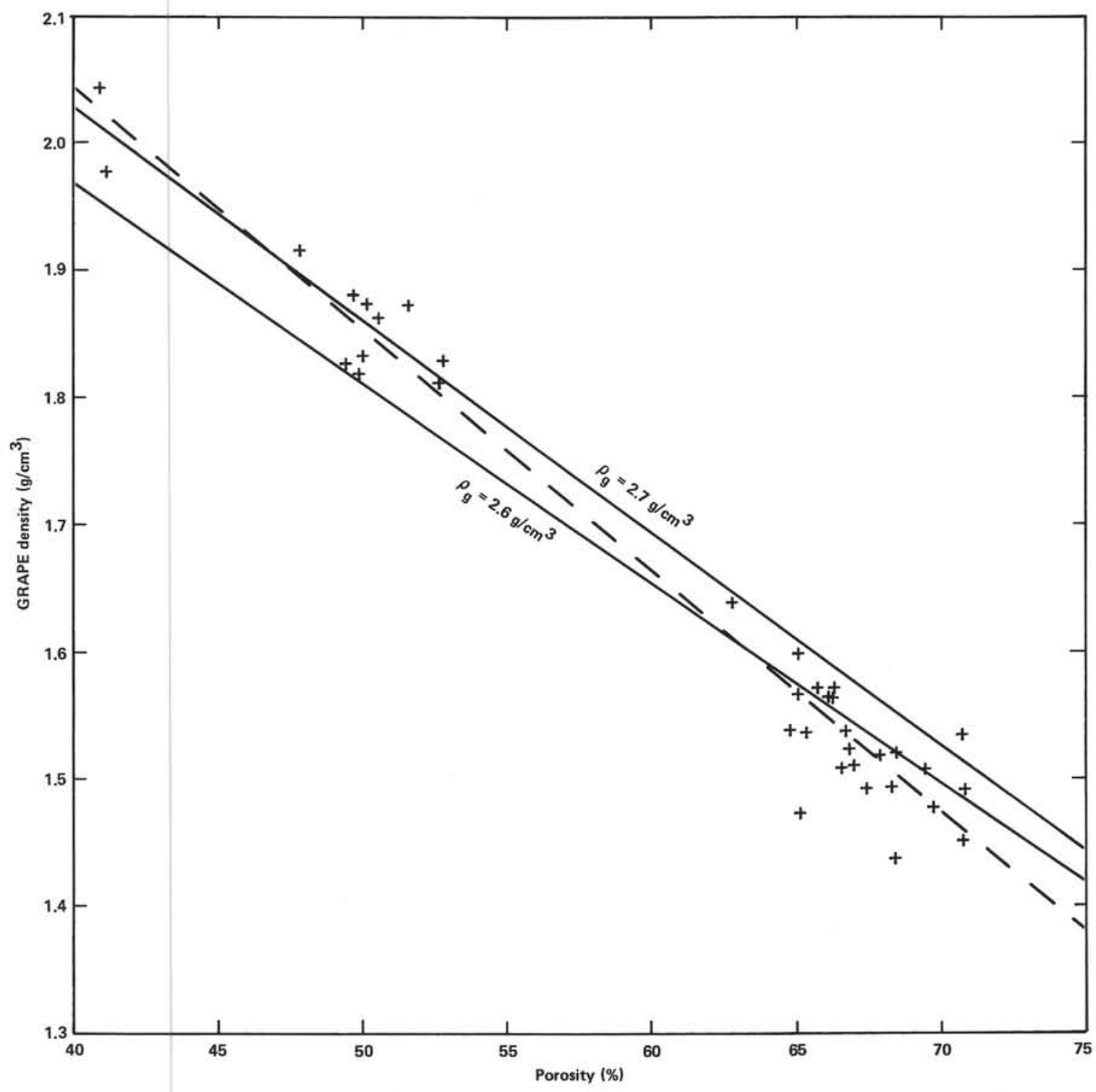

Figure 2. Hole 515B, GRAPE wet-bulk density versus gravimetric porosity. The dashed line shows the best-fitting linear relation determined by least squares (see Table 3 ). The solid lines show the theoretical relationships for completely saturated sediments. $\varrho_{\mathrm{b}}=\varrho_{\mathrm{f}} \phi+(1-\phi) \varrho_{\mathrm{g}}$ with a pore-fluid density of $1.025 \mathrm{~g} / \mathrm{cm}^{3}$ and grain densities as indicated.

Table 3. Summary of regression parameters.

\begin{tabular}{|c|c|c|c|c|c|c|c|}
\hline \multirow[b]{2}{*}{ Hole } & \multirow[b]{2}{*}{ Figure } & \multirow[b]{2}{*}{ Relation } & \multicolumn{3}{|c|}{ Parameters } & \multirow{2}{*}{$\begin{array}{c}\text { Number } \\
\text { of } \\
\text { samples }\end{array}$} & \multirow[b]{2}{*}{ r } \\
\hline & & & a & b & c & & \\
\hline $515 \mathrm{~B}$ & 1 & $e_{b}(g r a v)=.a+b \phi$ & $2.86 \pm 0.05$ & $-1.94 \pm 0.08$ & & 38 & 0.97 \\
\hline $515 \mathrm{~B}$ & 2 & $\mathrm{Qb}_{\mathrm{b}}(\mathrm{GRAPE})=\mathrm{a}+\mathrm{b} \phi$ & $2.81 \pm 0.04$ & $-1.90 \pm 0.06$ & & 36 & 0.98 \\
\hline $516 \mathrm{~F}$ & 3 & $\varrho_{b}($ grav. $)=a+b \phi$ & $2.73 \pm 0.01$ & $-1.75 \pm 0.02$ & & 242 & 0.99 \\
\hline $516 \mathrm{~F}$ & 4 & $e_{b}($ GRAPE $)=a+b \phi$ & $2.74 \pm 0.01$ & $-1.82 \pm 0.03$ & & 232 & 0.99 \\
\hline $516 \mathrm{~F}$ & 5 & $\varrho \mathrm{b}=\mathrm{a} \overrightarrow{V^{b}}+\mathrm{c}$ & $-2.15 \pm 0.15$ & $-1.74 \pm 0.25$ & $2.67 \pm 0.06$ & 205 & \\
\hline
\end{tabular}

Note: $\varrho_{b}$ is wet-bulk density; grav. is gravimetric; $\phi$ is porosity $(\%)$; $\bar{V}$ is mean compressional-wave velocity. 


\section{H. SCHAFTENAAR, R. P. MOORE, R. L. CARLSON}

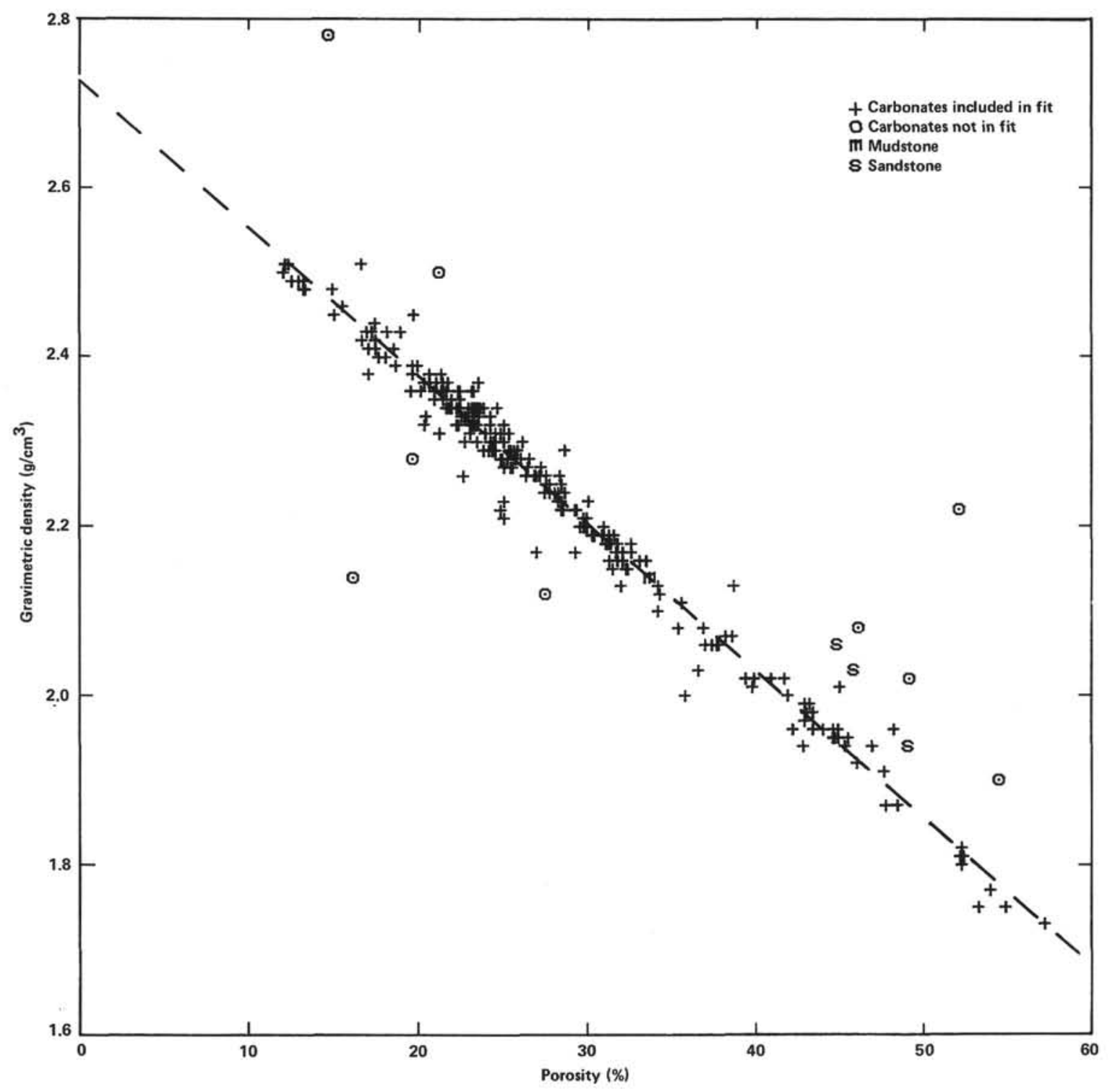

Figure 3. Hole 516F, gravimetric wet-bulk density versus gravimetric porosity. The dashed line shows the best-fitting linear relation determined by least squares (see Table 3). Clastics and indicated carbonates are not included in the linear regression. 


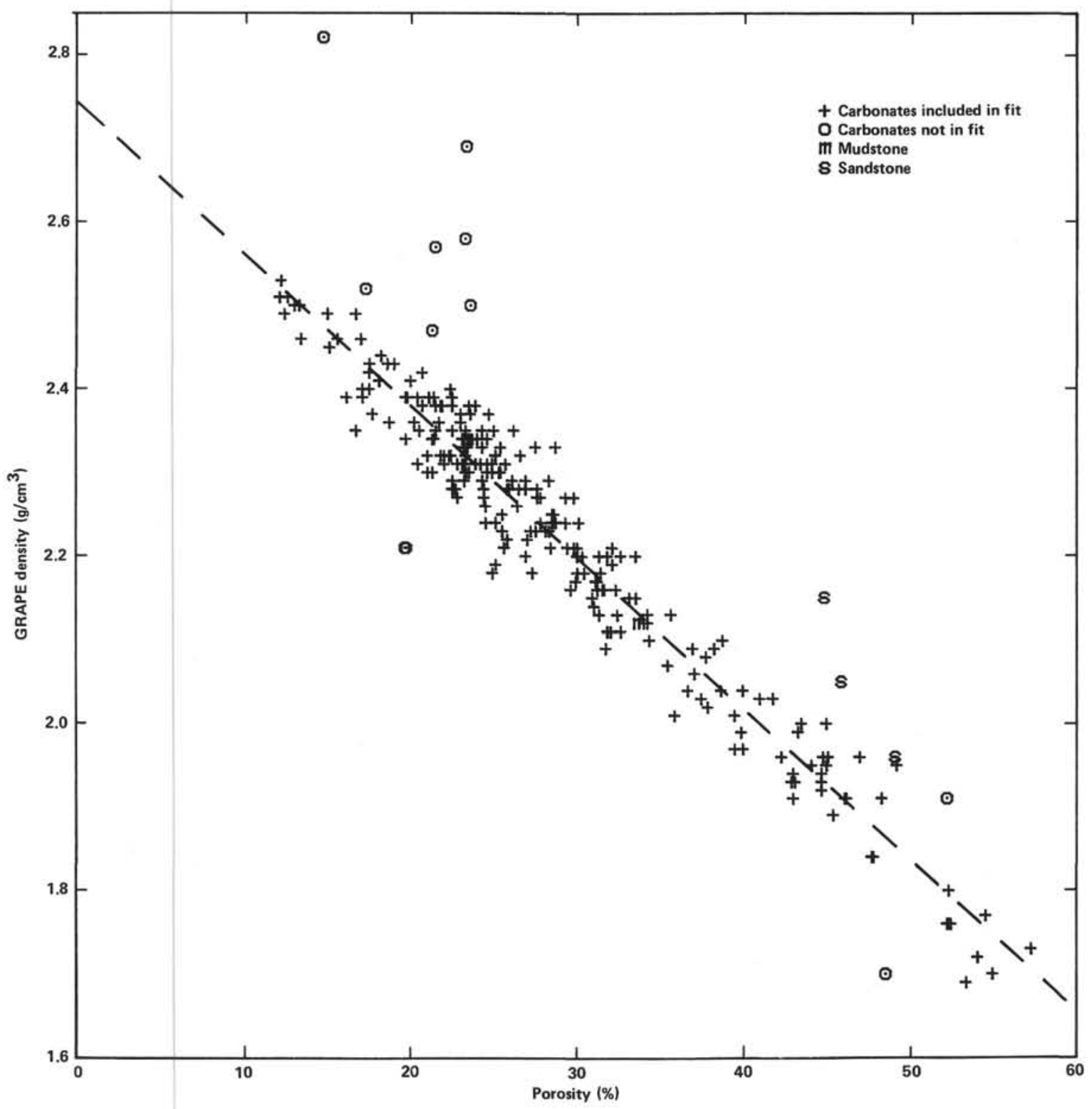

Figure 4. Hole 516F, GRAPE wet-bulk density versus gravimetric porosity. The dashed line shows the best-fitting linear relation determined by least squares (see Table 3). Clastics and indicated carbonates are not included in the linear regression. 
C. H. SCHAFTENAAR, R. P. MOORE, R. L. CARLSON

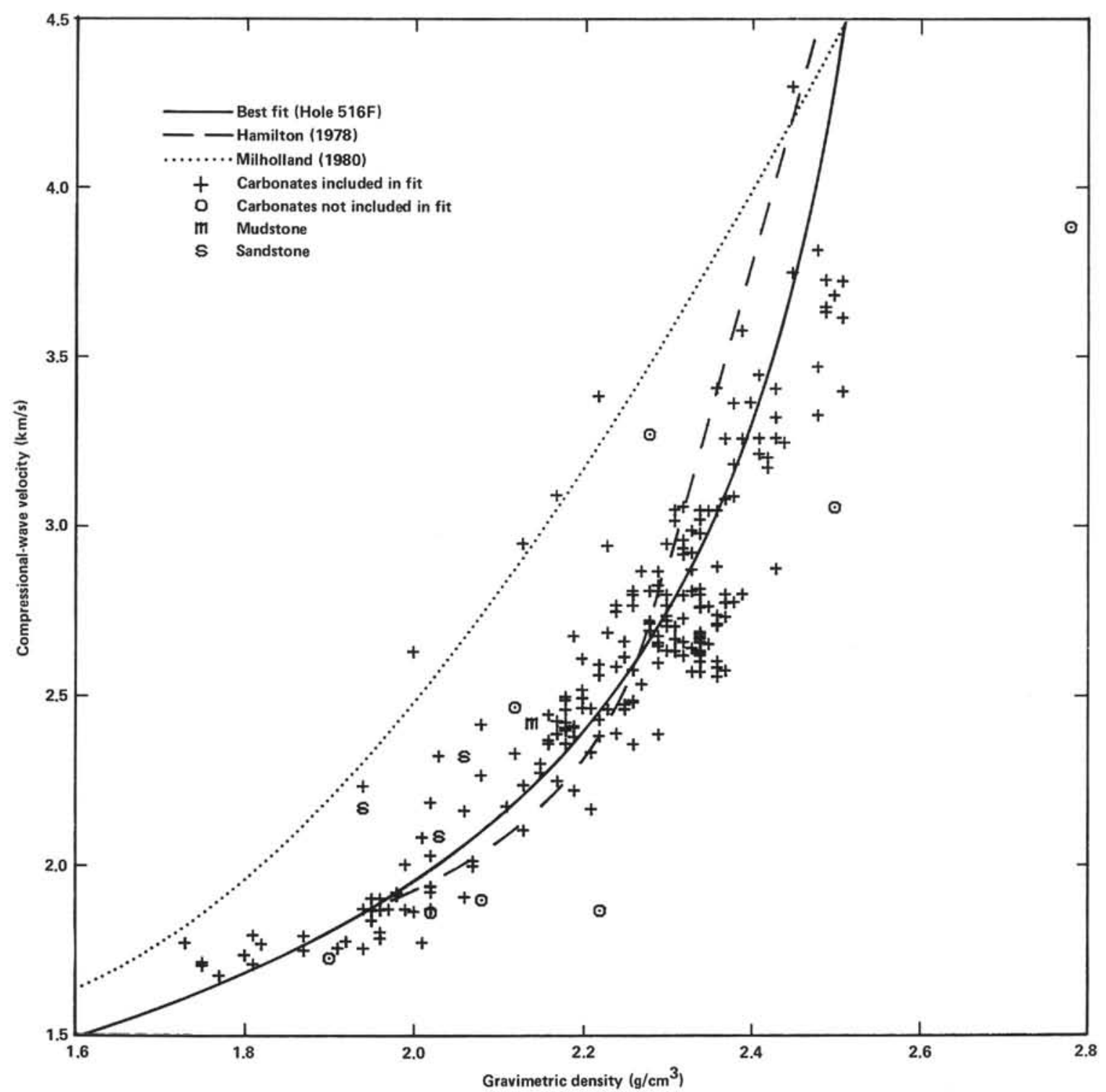

Figure 5. Hole 516F, compressional-wave velocity versus gravimetric wet-bulk density. The best-fitting relation of the form $\varrho_{\mathrm{b}}=\mathrm{a} \overrightarrow{V^{b}}+\mathrm{c}$ determined by least squares (see Table 3 ) and the empirical relations of Hamilton (1978) and Milholland (1980) are as indicated. 